\title{
A National Audit of the Management Pathways for New HIV Diagnoses
}

Kaide $\mathrm{E}^{1}$, Curtis $\mathrm{H}^{2}$, Freedman $\mathrm{A}^{3}$, Croxford $\mathrm{S}^{4}$, Burns $\mathrm{F}^{5,6}$, Sabin $\mathrm{CA}^{5,7}$, Chadwick $\mathrm{D}^{8}$, Sullivan $\mathrm{AK}^{9}$ on behalf of BHIVA Audit and Standards Subcommittee.

\section{Affiliations}

1. Genitourinary Medicine Department, Royal Liverpool and Broadgreen University Hospitals NHS Trust, UK

2. British HIV Association, London, UK

3. Cardiff University School of Medicine/University Hospital of Wales, Cardiff, UK

4. Public Health England (PHE) Blood Safety, Hepatitis, Sexually Transmitted Infections and HIV Division National Infection Service, UK

5. Institute for Global Health, University College London, London, UK

6. Royal Free London NHS Foundation Trust, London UK

7. National Institute for Health Research (NIHR) Health Protection Research Unit (HPRU) in Blood Borne and Sexually Transmitted Infections at UCL, UK

8. Centre for Clinical Infection, James Cook University Hospital, Middlesbrough, UK

9. Chelsea and Westminster Hospital NHS Foundation Trust, London, UK

\section{Keywords}

Europe, ART (antiretroviral therapy), combination ART (cART), Diagnosis, HIV (Human immunodeficiency virus) 


\section{Introduction}

In 2017, the United Kingdom (UK) met the UNAIDS 90:90:90 target to have over 90\% of those living with HIV being diagnosed (92\%), over $90 \%$ of those diagnosed being on antiretroviral therapy (ART, 98\%), and over $90 \%$ of those on ART having an undetectable viral load $(97 \%) .{ }^{1}$ This demonstrates the significant progress the UK has made in HIV care since the identification of the virus in $1983 .^{2}$ Despite this, HIV remains a stigmatised, socially complex and often traumatic diagnosis. ${ }^{3}$

The British HIV Association (BHIVA) Standards of Care for People Living with HIV provide guidance to support the delivery of quality HIV care nationally. Since 2013, these Standards have recommended that people newly diagnosed with HIV be fully assessed by an HIV specialist clinician within two weeks of receiving a positive HIV test result (target: $>90 \%) .{ }^{4}$ Timely review is not only beneficial to the clinical outcomes of individuals newly diagnosed with HIV, but also has the added advantage of minimising the risk of onward transmission through patient education, partner notification, and if ART is commenced, through treatment as prevention (TasP). ${ }^{5}$

The BHIVA guidelines for the treatment of HIV-1-positive adults with ART (2015) recommend that 'people with HIV start ART', immediately if the person is diagnosed during primary HIV infection, and within two weeks of commencing antimicrobials in those who are diagnosed with a CD4 count $<200$ cells $/ \mathrm{mm}^{3}$ and either an AIDS-defining infection or serious bacterial infection. For those not meeting these criteria, prompt ART initiation should be encouraged but should ultimately be guided by the individual's readiness to start. ${ }^{6}$ Notably, 
the NHS England Quality Dashboard uses the proportion of newly diagnosed adults who start ART within 3 months (91 days) of diagnosis, as one of series of surrogate markers for quality of care. $^{7}$

The purpose of this audit was to review the management of those newly diagnosed with HIV in the UK using the BHIVA Standards of Care as a benchmark for best practice. Our primary outcomes were to assess the time from initial positive/reactive test to 1) assessment by an HIV specialist clinician and 2) ART initiation. Our secondary outcomes were to explore the topics discussed at initial HIV clinician specialist review and the documented reasons for any delays in ART initiation.

\section{Methods}

UK specialist HIV services were invited to retrospectively review case notes of the last 40 adults (aged 16 years and over), assessed as newly diagnosed with HIV at their centres, between 1 January 2018 and 31 March 2019, or all such individuals if fewer than 40 . Responses were submitted electronically via a dynamic online web-based questionnaire (LimeSurvey GmbH, Hamburg, Germany), with each service being identified via a unique code.

Data requested were: demographics; probable HIV exposure category; presence or absence of disabilities or language difficulties; type of initial positive/reactive HIV test (laboratory-based or point of care) and setting where sample was taken; dates of testing, first being seen by an HIV specialist clinician following positive/reactive test, first discussion of ART initiation, 
and ART initiation, which were used to calculate intervals and then group into timeframes; whether specified topics had been covered at or before first discussion of ART initiation; and the reasons for any delay in ART initiation, if applicable, which respondents could select from various listed options with free text space also provided.

As this is a clinical audit, ethical approval was not required. Data were analysed in Microsoft ${ }^{\circledR}$ Excel 2010 (Microsoft Corporation, Redmond, Washington USA) using numbers and percentages for categorical data and medians and interquartile (IQR) ranges for numerical data. Results were based on summary statistics from each clinic. Median calculations were made based on an event having occurred, rather than using a Kaplan-Meier approach.

\section{Results}

Patient characteristics

Among 186 services thought to be providers of specialist HIV care, 132 provided data for 2281 eligible individuals for this audit. Of these services; 49 each submitted 1-9 cases, 37 each submitted 10-19 cases, 30 each submitted 20-39 cases and 16 each submitted $\geq 40$ cases. Three additional services reported that they had not assessed any eligible newly diagnosed individuals during the audit period.

As shown in table 1, compared to new HIV diagnoses reported in UK national surveillance data, ${ }^{8}$ the audited population included higher proportions of individuals who were aged 50 or 
over $(24.5 \%$ vs $21.3 \%)$, of white ethnicity $(60.0 \%$ vs $48.2 \%)$ and with heterosexual exposure to HIV (41.6\% vs 35.2\%). There was a higher proportion of missing data ('not available') for 'ethnicity' and 'exposure' characteristics in the UK national surveillance data when compared with the audited population.

[Insert Table 1]

Test settings

The settings in which the initial positive/reactive HIV specimens were taken, in descending order, were: 968 (42.4\%) genitourinary medicine (GUM)/sexual health or HIV clinic settings; 319 (14\%) inpatient settings; 289 (12.7\%) general practice; $273(12 \%)$ other outpatient clinics, including antenatal clinics; 100 (4.4\%) self-sampling or self-testing at home; 84 (3.7\%) emergency departments; 66 (2.9\%) 'other', which were mainly abroad or for visa purposes $(n=24)$, prison or immigration $(n=13)$, private or occupational health service $(n=12)$, blood donor service (n=6); $56(2.5 \%)$ acute medical unit (AMU); and $56(2.5 \%)$ community/voluntary organisations or outreach settings. Seventy $(3.1 \%)$ cases were submitted as 'not known/answered'.

Time from initial positive/reactive HIV test to first HIV specialist clinician review

Over two-thirds $(\mathrm{n}=1544,67.7 \%)$ of newly diagnosed patients were seen by an HIV specialist 
clinician with two weeks of receiving their test result. This increased to $83.1 \%(n=1896)$ within four weeks and $88.6 \%(n=2022)$ within 6 weeks, although the time interval varied widely between participating clinical services (Figure 1).

[Insert Figure 1]

The shortest times to specialist review were when testing was undertaken in an AMU or an inpatient setting, both of which demonstrated narrow interquartile ranges around a median of 4 days (Figure 2). Longer and more variable times to specialist review were seen where testing was initially undertaken in general practice (median 10 days) and non-sexual health, outpatient settings (median 9 days). However, for all test settings, a minority of individuals took much longer to be seen, with particularly long delays of 50 days and longer for those tested in general practice or in home settings (either self-testing or self-sampling).

[Insert Figure 2]

Time in weeks from initial positive/ reactive HIV test to ART initiation

Almost half ( $n=1099,48.2 \%)$ of newly diagnosed patients commenced ART within four weeks of their initial diagnosis (Figure 3). This increased to three-quarters $(n=1700,74.5 \%)$ at 8 weeks and $83 \%(n=1894)$ at 13 weeks. By the time of data collection, $141(6.2 \%)$ patients had not initiated ART at the clinical service where they were initially assessed. 
[Insert Figure 3]

Reasons for delay in ART initiation

Among individuals starting ART, the proportion with reported reasons for delay in doing so increased with the actual time from diagnosis to ART initiation (Figure 4). For those who initiated ART within 8 weeks, delays were predominantly due to either clinical reasons i.e. concomitant illness/medication or service factors i.e. time to receive test results or the need for virtual clinic approval for a non-standard regimen. Among those who initiated ART more than eight weeks after diagnosis, delays were more likely to be attributed to the individual not wishing to start ART when first offered, postponing or re-scheduling appointments, or not attending for booked appointments although clinical reasons remained important.

[Insert Figure 4]

Topics documented as covered at (or before) the first discussion of ART initiation

The benefits of ART were documented as discussed with $85.8 \%(\mathrm{n}=1957)$ of individuals (median 92\%, IQR 79-100) at, or before first discussion of ART initiation. Similarly, partner notification was discussed with $85.7 \%$ ( $\mathrm{n}=1954)$ of individuals (median 93\%, IQR 83-100). Documentation of discussions regarding the availability of peer or community support, and 
the evidence that viral suppression on ART means a person cannot sexually transmit HIV ("undetectable equals untransmittable", $\mathrm{U}=\mathrm{U})$ was less frequent; $61.4 \%(\mathrm{n}=1401)$ and $56.6 \%$ $(n=1290)$ respectively. There was wide variation between participating clinical services in the proportion of individuals with whom the latter two topics were discussed; this was evidenced by an IQR of $44-86 \%$ (median $65 \%$ ) for discussions regarding the availability of peer or community support and an IQR of 35-84\% (median 56\%) for ' $U=U$ ' discussions.

\section{Discussion}

This clinical audit of the routine management of adults newly diagnosed with HIV showed substantial variation between participating services in the times from the initial positive/reactive HIV test, to being seen by a specialist clinician and to ART initiation, and in the topics discussed at the first discussion of ART initiation. Reassuringly, in line with the BHIVA Standards of Care, the majority of individuals were reviewed by a specialist clinician within two weeks of a positive/reactive result (67.7\%) and were subsequently commenced on ART within 13 weeks of diagnosis (83\%); the latter corresponding to the NHS England Quality Dashboard measure.,

The largest proportion of positive/reactive HIV tests originated from the GUM/Sexual Health or HIV clinic setting (42.4\%), however, it did not follow that these individuals were seen by an HIV specialist clinician most promptly. This was the attainment of the inpatient setting, accounting for the second highest proportion of positive/reactive HIV tests (14\%), and where newly diagnosed individuals were reviewed within a median of four days (IQR 1-9 days) from diagnosis, compared with a median of 8 days (IQR 3-15 days) in the GUM/Sexual 
Health and HIV setting. The shorter time to HIV specialist clinician review in the inpatient setting potentially reflects individual clinical need and readily accessible internal referral pathways. ${ }^{9}$ Conversely, individuals tested in general practice $(12.7 \%)$ and 'other' outpatient clinics $(12 \%)$ had the longest median wait to be reviewed by an HIV specialist clinician; within 10 days (IQR 5-21 days) and 9 days (IQR 4-21 days) respectively.

Concerningly, although the median days from positive/reactive HIV test to an HIV specialist clinician review for all test settings was within the BHIVA recommended time interval, a minority of individuals tested in general practice and in home settings experienced an unacceptable wait of 50 days or longer before being reviewed. This result demonstrates the importance of ensuring a seamless and rapid transition from test setting into specialist care. This is especially important for individuals tested outside of HIV-aligned services, who may be less likely to be expecting an HIV diagnosis, which has a been shown to have a negative impact on readiness to commence ART. ${ }^{10,11}$

The time from initial positive/reactive test to commencing ART is intrinsically dependent on the time from test to first HIV specialist review, as in the UK the former is a prerequisite for the latter. In this audit population, $17.5 \%$ of individuals commenced ART at initial specialist review and a further $53.7 \%$ did so within the subsequent 28 days, thus demonstrating the benefit of early linkage into specialist care. The exact timing of ART initiation should be guided by both clinical presentation and individual readiness to commence ART ('ART readiness'). ${ }^{10,11}$ Soon after diagnosis, delays in ART initiation were primarily attributed to 'clinical reasons' i.e. concurrent opportunistic illnesses and infection, although overall those with primary or advanced HIV were more likely to commence ART sooner (data not shown), 
in line with BHIVA guidance. With increasing time from positive/reactive test, delays in commencing ART reflected individuals initially declining ART, postponing or not attending appointments; these factors potentially serve as surrogate markers for ART readiness, especially in those initially declining, and demonstrate the importance of reviewing individuals who have not commenced ART within 8 weeks of diagnosis, in order to offer additional support. ${ }^{11}$

ART readiness is a complex concept being influenced by, amongst other things, an individual's understanding of HIV infection and the benefits of ART, and according to Gebrekristos et al., the 'drive to live', which encompasses psychological factors such as shame, stigma, fear of death and protective factors such as social support and personal resilience. ${ }^{11}$ The benefits of ART were documented as being discussed with the majority of individuals at, or before, the first discussion of ART initiation.

In contrast, discussions regarding the availability of peer and community support were variable. This could potentially reflect discrepancies in the provision of these services nationally, ${ }^{12}$ but may also reflect a historical emphasis on physical health over mental wellbeing in medical practice. ${ }^{13}$ Despite the knowledge that an undetectable viral load on ART renders HIV sexually untransmittable $(\mathrm{U}=\mathrm{U}),{ }^{14}$ this topic was only documented as being discussed with $56 \%$ of individuals, again with significant between-clinic variation. However, the proportion of these discussions did increase over the audit period, possibly reflecting increasing clinician confidence in conveying this message following its international endorsement (data not shown). ${ }^{15,16}$ It is crucial that efforts are made to ensure topics advised in the BHIVA Standards of Care are delivered to newly diagnosed individuals. 


\section{Limitations}

The findings of this audit may have been impacted by data which was not available to us, this includes information from HIV services that did not engage with the audit process and information from clinician-patient discussions which were not documented in the case notes. As our data was solely submitted from specialist HIV services, individuals tested but not linked into care would not be represented. Based on surveillance of laboratory tests, $94 \%$ of adults newly diagnosed with HIV in the UK in 2017 were linked to HIV care within three months ${ }^{17}$, but there may also be undisclosed reactive self-tests undertaken in home settings. In addition, although the data is illustrative of the United Kingdom, it is not representative because each HIV service was asked to submit data for 40 individuals which represents a relative under-sampling from larger clinics.

\section{Conclusion}

Overall, the findings of this audit are positive, demonstrating that the majority of individuals newly diagnosed with HIV are reviewed by a specialist clinician and commenced on ART promptly. However, the findings also suggest a need for more streamlined pathways into HIV care, especially for those diagnosed outside of GUM/Sexual Health/HIV and inpatient settings; review of individuals who have not commenced ART within 8 weeks of diagnosis to identify possible support needs, and the development of protocols to ensure relevant topics are discussed with all newly diagnosed individuals. 


\section{Members of BHIVA Audit and Standards Subcommittee}

V Balasubramaniam, F Burns, D Chadwick (chair), E Cheserem, D Churchill, S Croxford, A Freedman, E Kaide, P Khan, R Kulasegaram, N Larbalestier, R Mbewe, O Olarinde, E Ong, S Pires, R Raya, C Sabin, A Sullivan (vice-chair).

\section{Acknowledgements}

The research was supported, in part, by the National Institute for Health Research Health Protection Research Unit (NIHR HPRU) in Blood Borne and Sexually Transmitted Infections at University College London in partnership with Public Health England (PHE), in collaboration with London School of Hygiene \& Tropical Medicine (LSHTM). The views expressed are those of the authors and not necessarily those of the NIHR, the Department of Health and Social Care or Public Health England.

Participating clinical services: Aberdeen, Woolmanhill Hospital; Airdrie, Monklands Hospital; Ashton-under-Lyne, Ashton Primary Care Centre; Barking, Barking Community Hospital; Barnsley, Barnsley Hospital NHS Foundation Trust; Barnstaple, North Devon District Hospital; Basingstoke, Solent NHS Trust; Bath, Royal United Hospital; Bedford, iCASH Bedfordshire; Belfast, Royal Hospitals Trust; Birmingham, Birmingham Heartlands Hospital; Birmingham, Queen Elizabeth Hospital; Bishop Auckland, Bishop Auckland General Hospital; Bournemouth, Royal Bournemouth Hospital; Bradford, Bradford Hospitals 
NHS Trust; Brighton, Royal Sussex County Hospital; Burton-upon-Trent, Queen's Hospital; Bury St Edmunds, West Suffolk Hospital; Cambridge, Addenbrooke's Hospital NHS Trust; Canterbury, Kent and Canterbury Hospital; Cardiff, Cardiff Royal Infirmary; Cardiff, University Hospital of Wales; Carshalton, St Helier Hospital; Chatham, Medway Sexual Health; Chelmsford, Mid Essex Hospital Services NHS Trust; Chester, Fountains Building; Chesterfield, Chesterfield Royal Hospital NHS Foundation Trust; Chichester, St Richard's Hospital; Cottingham, Castle Hill Hospital; Coventry, City of Coventry Health Centre; Crewe, Leighton Hospital; Croydon, Croydon University Hospital; Darlington, Darlington Memorial Hospital; Derby, Derbyshire Royal Infirmary NHS Trust; Doncaster, Doncaster Royal Infirmary; Dundee, Ninewells Hospital and Medical School; Durham, University Hospital of North Durham; Eastbourne, Avenue House Sexual Health Clinic; Edinburgh, Lothian University Hospitals; Edinburgh, Western General Hospital; Exeter, Exeter NHS Walk-in Centre; Galashiels, Borders Sexual Health; Glasgow, Gartnaval General Hospital; Great Yarmouth, Northgate Hospital; Guildford, Buryfields Clinic; Halifax, Sexual Health Centre; Harrow, Northwick Park Hospital; Hastings, Station Plaza Health Centre; Hereford, Wye Valley NHS Trust; High Wycombe, Wycombe General Hospital; Huntingdon, The Oak Tree Centre; Inverness, Raigmore Hospital; Ipswich, Orwell Clinic; Kettering, St Mary's Hospital; Kidderminster, Worcestershire Acute Hospitals NHS Trust; King's Lynn, iCASH Norfolk; Kingston upon Thames, Kingston Hospital; Kirkcaldy, Whytemans Brae Hospital; Leeds, Leeds Teaching Hospitals NHS Trust; Lincoln, Lincolnshire Community Health Services NHS Trust; Liverpool, Royal Liverpool University Hospital; Llantrisant, Royal Glamorgan Hospital; London, 10 Hammersmith Broadway; London, Central Middlesex Hospital; London, Chelsea and Westminster Hospital; London, Chelsea and Westminster Hospital; London, Homerton University Hospital NHS Foundation Trust; London, Imperial College Healthcare NHS Trust; London, King's College Hospital; London, Mortimer Market 
Centre; London, Newham University Hospital; London, North Middlesex Hospital; London, Queen Elizabeth Hospital; London, Royal Free London NHS Foundation Trust; London, Sir Ludwig Guttman Health and Wellbeing Centre; London, St George's Hospital; London, St Thomas' Hospital; London, The Royal London Hospital; London, University Hospital Lewisham; Londonderry, Altnagelvin Area Hospital; Luton, Luton \& Dunstable Hospital NHS Trust; Macclesfield, Macclesfield District Hospital; Maidstone, Maidstone Hospital; Manchester, Manchester Royal Infirmary; Manchester, North Manchester General Hospital; Manchester, Withington Hospital; Middlesbrough, James Cook University Hospital; Milton Keynes, Milton Keynes General Hospital; Newcastle upon Tyne, New Croft Sexual Health Centre; Newcastle upon Tyne, Royal Victoria Infirmary; Newport, Royal Gwent Hospital; Norwich, Norfolk \& Norwich University Hospital; Nottingham, Nottingham City Hospital; Nuneaton, George Eliot Hospital NHS Trust; Oxford, Oxford Radcliffe NHS Trust; Peterborough, Peterborough and Stamford NHS Foundation Trust; Portsmouth, St Mary's Hospital; Reading, Royal Berkshire Hospital; Rhyl, Glan Clwyd District General Hospital NHS Trust; Rotherham, Rotherham NHS Foundation Trust; Rugby, Hospital of St Cross; Salford, Lance Burn Sexual Health Centre; Salisbury, Salisbury District Hospital; Sheffield, Royal Hallamshire Hospital; Shiremoor, One to One Centre; Shrewsbury, Royal Shrewsbury Hospital; Slough, Upton Hospital; Southall, Ealing Hospital; Southampton, Royal South Hants Hospital; Southport, Southport \& Ormskirk NHS Trust; St Helens, St Helen's and Knowsley Hospital; Stevenage, Southgate Health Centre; Stockport, Manchester Foundation NHS Trust; Stratford-upon-Avon, Stratford Hospital; Sunderland, Sunderland Royal Hospital; Sutton-in-Ashfield, Sherwood Forest Hospitals NHS Foundation Trust; Swansea, Singleton Hospital; Swindon, Great Western Hospitals NHS Foundation Trust; Taunton, Musgrove Park Hospital; Telford, Princess Royal Hospital NHS Trust; Torquay, Torbay Hospital; Truro, Royal Cornwall Hospital; Uxbridge, The Hillingdon Hospital; Walsall, 
Manor Hospital; West Bromwich, Sandwell District General Hospital; Westcliffe on Sea, Southend Hospital; Weymouth, Weymouth Community Hospital; Wirral, Arrowe Park Hospital; Wolverhampton, New Cross Hospital; Worksop, TriHealth Bassetlaw Integrated Sexual Health Service; Wrexham, Wrexham Maelor Hospital; York, York Hospitals NHS Trust.

\section{Declaration of Conflicting Interests}

FB has received funding for preparation of educational materials from Gilead Sciences. CS has received funding for membership of data safety and monitoring committees and participation in Advisory Boards. CS, DC and AF have received funding for preparation of educational materials, and DC for preparation of research projects, from Gilead Sciences and ViiV Healthcare. 


\section{References}

1. Kirby T. The UK reaches UNAIDS 90-90-90 targets. Lancet. 2018;392(10163):2427. doi:10.1016/S0140-6736(18)33117-9

2. Gallo RC, Montagnier L. The discovery of HIV as the cause of AIDS. $N$ Engl J Med. 2003;349(24):2283-2285. doi:10.1056/NEJMp038194

3. Walker L. 'There's no pill to help you deal with the guilt and shame': Contemporary experiences of HIV in the United Kingdom. Health (London). 2019;23(1):97-113. doi:10.1177/1363459317739436

4. British HIV Association. BHIVA Standards of Care for People Living with HIV 2013, https://www.bhiva.org/standards-of-care-2013_(2013, accessed 02 March 2020).

5. Montaner JS, Lima VD, Harrigan PR et al. Expansion of HAART coverage is associated with sustained decreases in HIV/AIDS morbidity, mortality and HIV transmission: The "HIV Treatment as Prevention" experience in a Canadian setting. PLoS One. 2014;9(2), e87872.

6. British HIV Association. The BHIVA guidelines for the treatment of HIV-1-positive adults with ART, https://www.bhiva.org/HIV-1-treatment-guidelines (2015, accessed 02 May 2020).

7. Public Health England. HIV Quality Dashboard 2018/2019, https://www.england.nhs.uk/wp-content/uploads/2018/03/hiv-metric-definitions2018-19-v2.pdf_(2018, accessed on 23 March 2020).

8. Public Health England. Trends in new HIV diagnoses and in people receiving HIVrelated care in the United Kingdom: data to the end of December 2018. Report, Health Protection Report Volume 13 Number 31, p.2-7, 2019. 
9. The King's Fund. Referral management: lessons for success, https://www.kingsfund.org.uk/sites/default/files/Referral-management-lessons-forsuccess-Candace-Imison-Chris-Naylor-Kings-Fund-August2010.pdf (2010, accessed on 16 March 2020).

10. Morgenstern T, Grimes D, Grimes R. Assessment of readiness to initiate antiretroviral therapy. HIV Clinical Trials. 2002; 3:2, 168-172.

11. Gebrekristos HT, Mlisana KP, Karim QA. Patients' readiness to start highly active antiretroviral treatment for HIV. BMJ. 2005;331(7519):772-775. doi:10.1136/bmj.331.7519.772

12. Terrence Higgins Trust. Centres and services, https://www.tht.org.uk/centres-andservices?category=26\&geolocation_geocoder_google_geocoding_api=\&geolocation_ geocoder_google_geocoding_api_state=1\&geocoding-lat=\&geocoding$\operatorname{lng}=\&$ geocoding $=100 \&$ page $=1(2020$, accessed on 04 June 2020).

13. The King's Fund. Transforming our healthcare system: Ten priorities for commissioners, https://www.kingsfund.org.uk/sites/default/files/field/field_publication_file/10Prioriti esFinal2.pdf (2015, accessed on 23 March 2020).

14. Rodger AJ, Cambiano V, Bruun T, et al. Risk of HIV transmission through condomless sex in serodifferent gay couples with the HIV-positive partner taking suppressive antiretroviral therapy (PARTNER): final results of a multicentre, prospective, observational study. Lancet. 2019;393(10189):2428-2438. doi:10.1016/S0140-6736(19)30418-0

15. Ngure K, Ongolly F, Dolla A, et al. "I just believe there is a risk" understanding of undetectable equals untransmissible $(\mathrm{U}=\mathrm{U})$ among health providers and HIV- 
negative partners in serodiscordant relationships in Kenya. J Int AIDS Soc. 2020;23(3):e25466. doi:10.1002/jia2.25466

16. Calabrese SK, Mayer KH. Providers should discuss $U=U$ with all patients living with HIV. Lancet HIV. 2019;6(4):e211-3.

17. O'Halloran C, Sun S, Nash S et al. HIV in the United Kingdom: Towards Zero 2030. 2019 report. December 2019, Public Health England, London. 


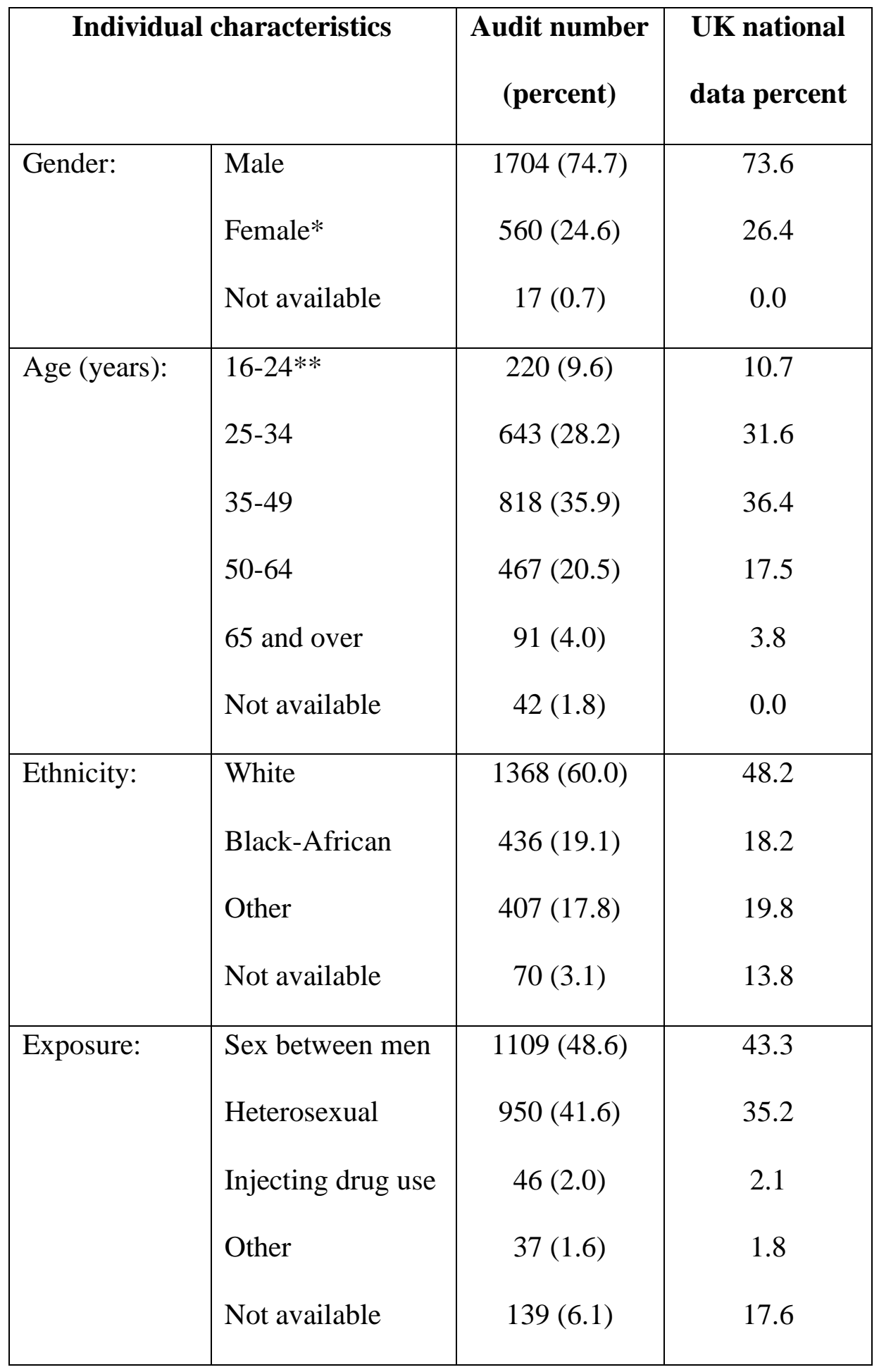

Table 1. Individual characteristics: audit population compared with UK national surveillance data for new diagnoses during 2018. *including 13 transwomen;** 15-24 in UK data. 


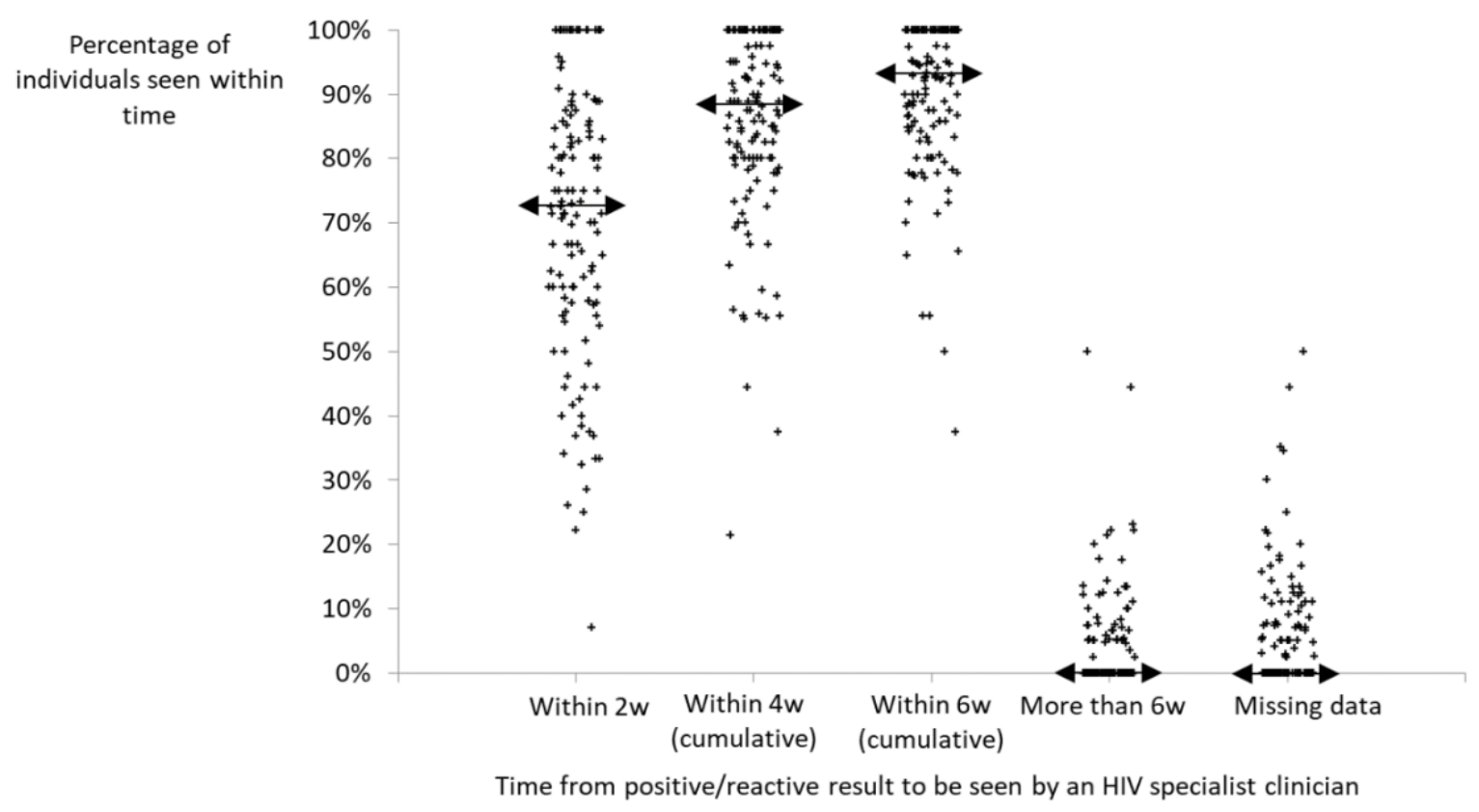

Figure 1: Variation between participating clinical services in time from initial positive/ reactive HIV test to first being seen by an HIV specialist clinician: each cross represents one service, with median across services indicated by a double-headed arrow. 


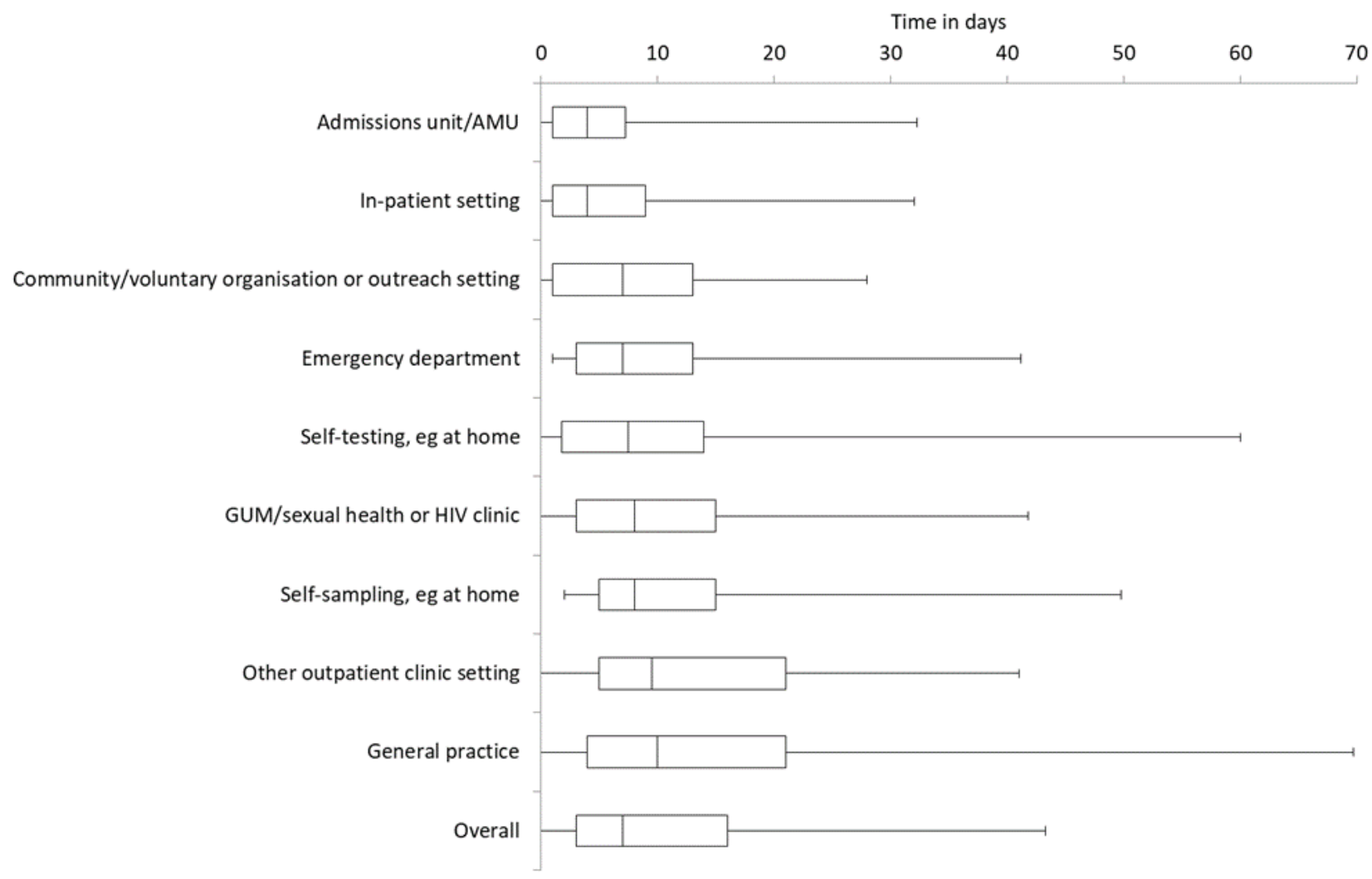

Figure 2: Time to first HIV specialist clinician review by setting in which the specimen was taken for the initial positive/ reactive HIV test (excluding missing data): boxes show upper and lower quartiles with median across, whiskers show $5^{\text {th }}$ and $95^{\text {th }}$ percentiles. 


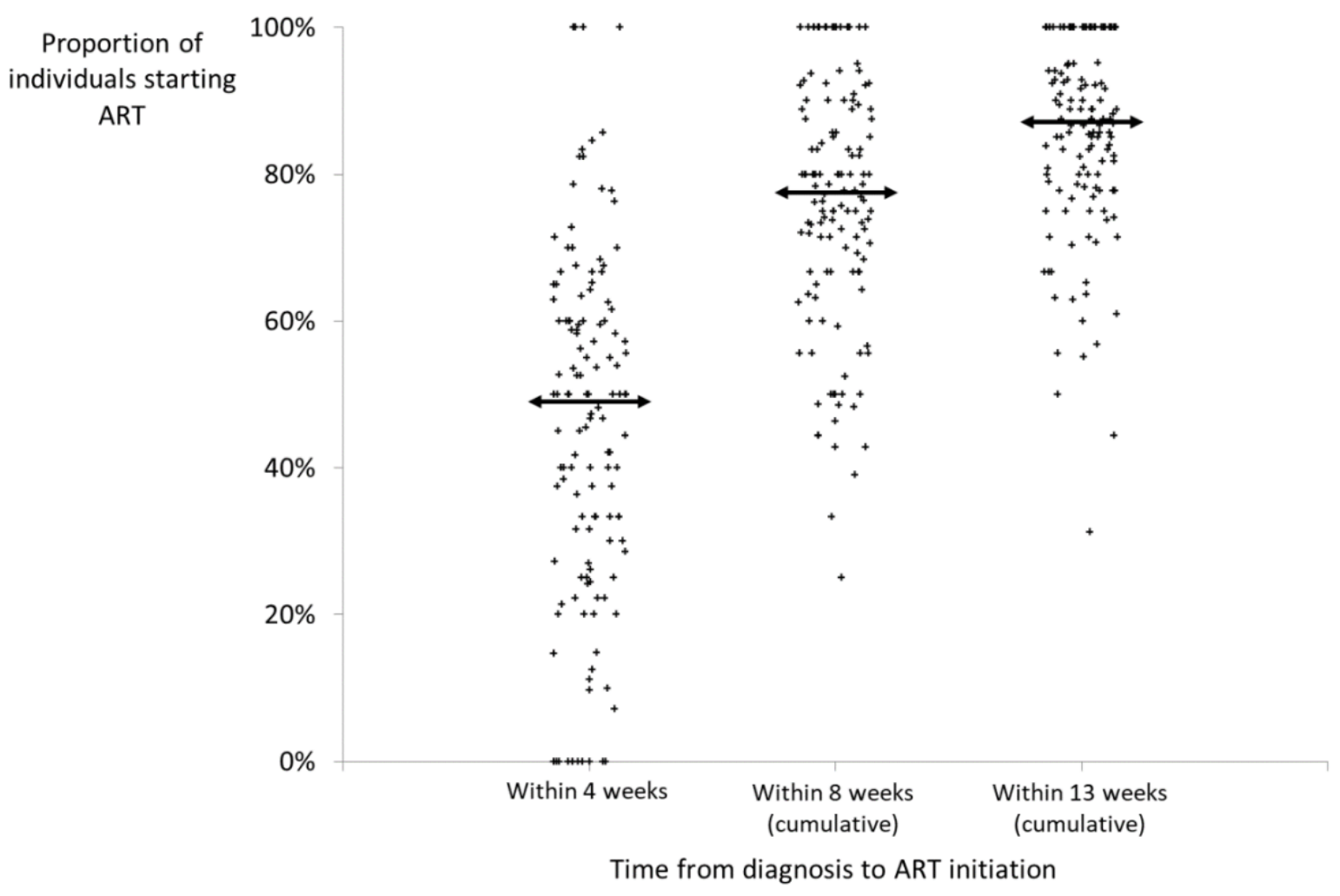

Figure 3: Variation between participating clinic services in time from diagnosis to ART initiation: each mark represents one service, with medians across services shown as horizontal bars. 


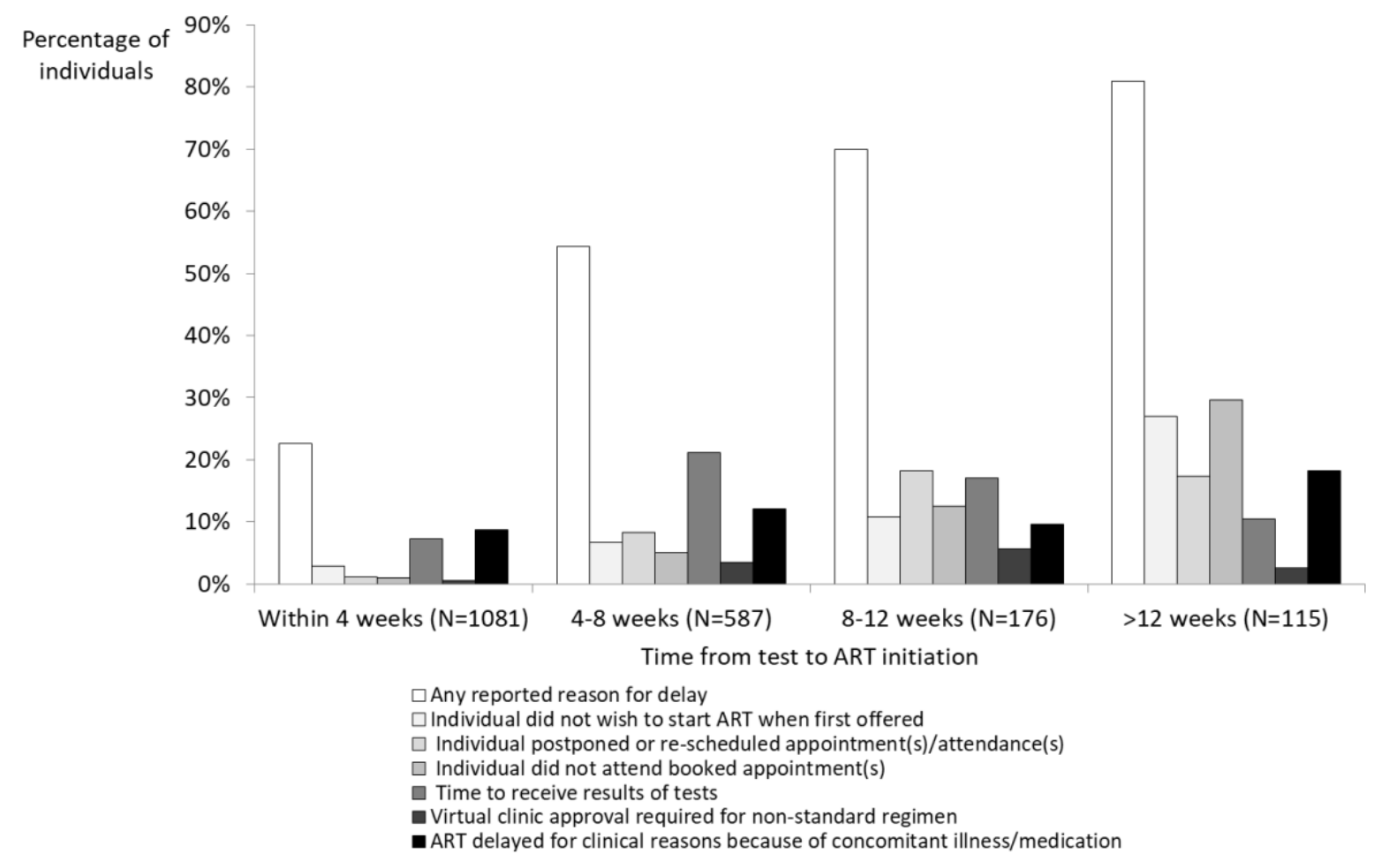

Figure 4. Proportion of individuals with reported reasons for delay in ART initation grouped by actual time to initation 